Gut, 1988, 29, 593-597

\title{
Cryptosporidial diarrhoea in AIDS and its treatment
}

\author{
G M CONNOLLY, M S DRYDEN, D C SHANSON, AND B G GAZZARD
}

From St Stephen's Hospital, London

\begin{abstract}
SUMMARY Of 234 patients with AIDS diagnosed at St Stephen's Hospital between January 1981 and June 1987, $26(11 \%)$ were found to have cryptosporidiosis. Stool examination was positive in all patients, but an average of three specimens (range 1-6) were required before a positive diagnosis was made. Other methods of diagnosis included jejunal and rectal biopsy and aspiration of the duodenal contents. Twenty three $(89 \%)$ lived for six months from the time of diagnosis and $16(60 \%)$ were alive at one year. Only five patients died as a direct result of cryptosporidial infection, while 10 other patients died from another complication of AIDS. Fifteen patients were enrolled in a prospective controlled study of erythromycin or spiramycin in the treatment of cryptosporidial diarrhoea. Most patients showed a significant response to antibiotic therapy but treatment was limited because of side effects. All patients responded to antidiarrhoeal agents, particularly long acting morphine sulphate. Three of our patients recently given zidovudine (AZT) have responded with a cessation of diarrhoea and cryptosporidia are no longer isolated from the stools.
\end{abstract}

Cryptosporidia are coccidian parasites which were recognised at the beginning of this century.' During the 1970 s veterinarians emphasised their importance as a cause of diarrhoea in animals. ${ }^{2}$ The first case in a human being was reported in 1976 when cryptosporidia were seen on a rectal biopsy specimen of a child with enterocolitis. ${ }^{3}$ There have been numerous reports since of infection in immunocompromised ${ }^{4}$ and immunocompetent patients where it has been recognised as a cause of travellers diarrhoea. ${ }^{5}$

The first reports of cryptosporidia causing diarrhoea in AIDS patients were received by the Centres for Disease Control (CDC) as early as 1979. By November 1982, 21 men had been reported to have developed the disease ${ }^{6}$ and since then the CDC has included in its definition of AIDS those patients who have intestinal cryptosporidiosis for longer than one month and in whom no other course for immunosuppression exists.

We report our experience with this infection at $\mathrm{St}$ Stephen's Hospital and its response to treatment in a group of patients with AIDS.

Address for correspondence: G M Connolly, MB MRCPI, St Stephen's Hospital, Fulham Road, London SW10 9TH.

Received for publication 16 November 1987.
Methods

PATIENTS

All patients with evidence of HIV infection and diarrhoea had at least six stool specimens examined. All specimens were examined by routine microbiological techniques to exclude infection with bacterial pathogens. Cryptosporidia were identified by a modified Ziehl Neelsen technique, ${ }^{7}$ both direct and concentrated. Rectal and duodenal biopsies were processed for examination by light microscopy in the usual way and stained with periodic acid Shiff's stain and Southgate's mucicarmine. Duodenal aspirates were examined in the same manner as the stools.

ANTIBIOTIC TREATMENT

Fifteen patients took part in a prospective controlled study with erythromycin or spiramycin in the treatment of diarrhoea. All were inpatients at the start of the study, although six were instructed on how to record accurate stool charts and were allowed to continue the treatment at home. They were randomly allocated either to erythromycin $500 \mathrm{mg}$ qds orally or spiramycin $500 \mathrm{mg}$ qds (in the ratio of 2:1) and stool charts were recorded accurately for 
Table 1 Pathogens isolated from AIDS patients with diarrhoea

\begin{tabular}{lr}
\hline Pathogen & $n$ \\
\hline Cryptosporidium & 26 \\
Mycobacterium avium-intracellulare & 9 \\
Entamoeba histolyctica & 8 \\
Giardia lamblia & 4 \\
Salmonella spp & 3 \\
Cytomegalovirus & 3 \\
Campylobacter & 2 \\
Shigella sp & 1 \\
\hline
\end{tabular}

that week. The patients were then given no therapy for a week although the stools were still collected, measured and examined bacteriologically. All antidiarrhoeals were withdrawn for this period of the study.

The stool volumes were monitored for a subsequent week on antidiarrhoeal therapy (a dose of diphenoxylate adjusted to the patient's convenience). A significant response was defined as a greater than $50 \%$ reduction of stool volume on treatment.

\section{Results}

\section{CLINICA L}

Of 234 patients who were HIV positive and had AIDS diagnosed at St Stephen's Hospital between 1981 and 1987, $61(26 \%)$ complained of persistent diarrhoea at presentation. Although a range of pathogens was seen (Table 1) the commonest was cryptosporidium. All but five patients had a positive diagnosis of a potential cause of diarrhoea as a result of stool cultures.

In 16 of the 26 patients with cryptosporidiosis this was the initial presentation of AIDS. In seven further patients cryptosporidiosis was diagnosed subsequent to an episode of Pneumocystis carinii pneumonia (PCP) and in three other patients Kaposi's sarcoma was the presenting complaint.

Six of the 16 patients who had AIDS diagnosed on the basis of cryptosporidial diarrhoea went on to develop other manifestations of AIDS over a 12 months follow up period. Fifteen patients had lost more than $5 \mathrm{~kg}$ in weight at presentation and this occurred in all patients during the course of their illness (Fig. 1).

Stool volumes ranged from $500 \mathrm{cc}$ to $3000 \mathrm{cc} / 24$ hours with frequencies from between 3-20 stools/day (Fig. 1). Two patients became constipated after the diagnosis without any medication, although cryptosporidium continued to be identified in the stools. The diarrhoea contained blood in one patient and mucus in another. Only six patients complained of significant abdominal pain at presentation. Vomiting
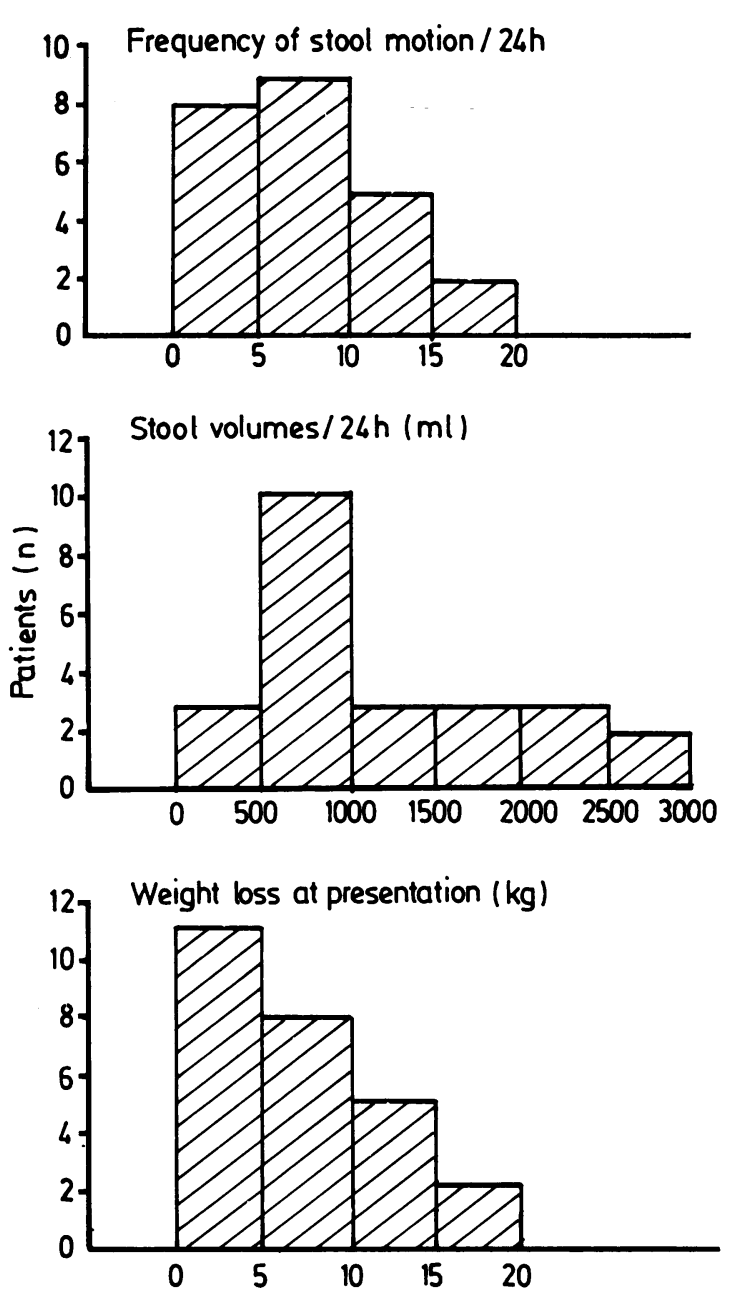

Fig. 1 Frequency and volume of stool per 24 hours and weight loss at presentation in AIDS patients with cryptosporidial diarrhoea

did not occur initially in any patient but was reported shortly before death in five patients, and could be related to another factor in two of these.

\section{DIAGNOSIS}

Stool samples were positive in all patients, but on average three specimens were required before a diagnosis was obtained. Ten patients had a duodenal biopsy and in seven cryptosporidia were seen in the mucosa and in the duodenal aspirate. All these patients, except one, also had cryptosporidia in the stools. This latter patient had six negative stool cultures but after the duodenal biopsy findings, a further six stool cultures were sent and one of these was positive for cryptosporidium. 
Table 2 Incidence of cryptosporidial diarrhoea (19811987)

\begin{tabular}{|c|c|c|c|}
\hline Year & Patients (n) & $\begin{array}{l}\text { Patients with } \\
\text { diarrhoea }(\%)\end{array}$ & $\begin{array}{l}\text { Patients with } \\
\text { cryptosporidiosis } \\
(\%)\end{array}$ \\
\hline 1981 & 1 & 0 & 0 \\
\hline 1982 & 1 & 1 & 0 \\
\hline 1983 & 3 & 0 & 0 \\
\hline 1984 & 16 & $3(20 \%)$ & 0 \\
\hline 1985 & 56 & $17(30 \%)$ & $3(17 \%)$ \\
\hline 1986 & 94 & $22(25 \%)$ & $14(63 \%)$ \\
\hline $1987^{*}$ & 63 & $18(25 \%)$ & $9(50 \%)$ \\
\hline
\end{tabular}

$*=6$ months

The proportion of AIDS patients with diarrhoea who were found to have cryptosporidiosis increased from 1981 to 1987 (Table 2) and the numbers of cases seen changed with the season (Fig. 2). Of 12 patients who had a rectal biopsy the diagnosis was established histologically in only two, both of whom had cryptosporidia on stool samples.

\section{RADIOLOGY}

The barium enema was normal in all nine patients examined. A barium follow through showed a nonspecific malabsorption pattern in two of seven patients. These two patients both had cryptosporidia seen on the jejunal biopsy and had an abnormal xylose and Schilling test.

\section{OU TCOME}

Five of the 26 patients have died of cryptosporidiosis (Fig. 3) over an average follow up period of 10 months (range 3-18). Three of these five had weight

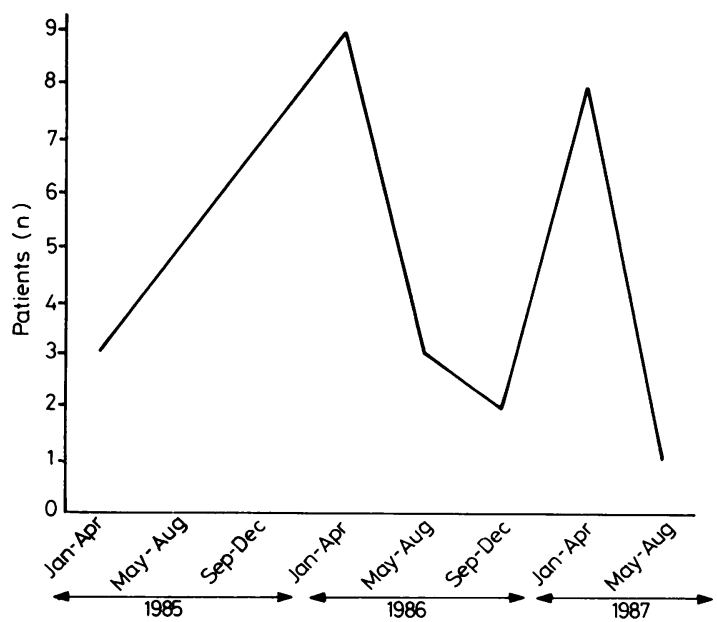

Fig. 2 Seasonal variation in diagnosis of cryptosporidial diarrhoea in AIDS.
$\Delta$ Alive

- Death from cryptosporidiosis

- Death from some other complication of AIDS

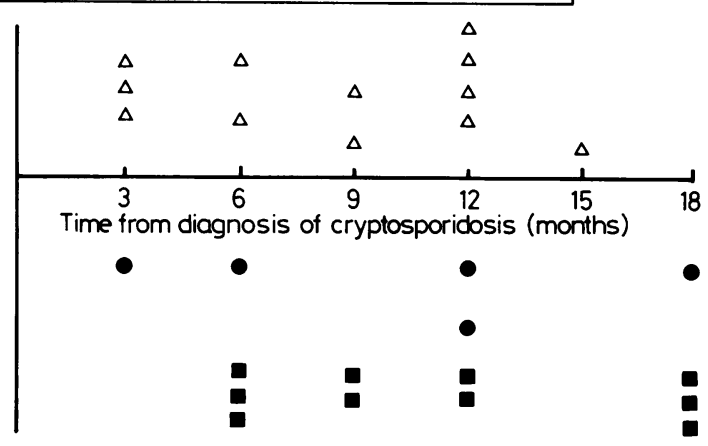

Fig. 3 Survival time with cryptosporidial diarrhoea in AIDS.

loss of more than $15 \mathrm{~kg}$ at presentation and stool volume of $3 \mathrm{l} / \mathrm{day}$. The two other patients who died a year or more after diagnosis had a lesser degree of weight loss, only moderate diarrhoea and led active independent lives taking antidiarrhoeal agents and antibiotics intermittently.

Over the same period of follow up 10 patients died from other complications of AIDS (Fig. 3). None of these patients had significant diarrhoea in the two months preceding their death. Two of these patients died of a severe wasting syndrome, but diarrhoea was not a complaint. One patient developed a toxic megacolon as a complication of cryptosporidiosis, ${ }^{8}$ but made an uneventful recovery without specific therapy and remained alive and well for nine months until he developed fatal $P$ carinii pneumonia.

TREATMENT

Seven of the 26 patients did not receive antibiotic therapy. Four were considered terminally ill, two as a direct result of cryptosporidial infection and two because of other AIDS complications. Two further patients developed spontaneous constipation which has continued despite the presence of cryptosporidia in the stools and one patient refused to attend follow up. Our initial experience of antibiotic therapy in nine patients indicated that erythromycin reduced the severity of diarrhoea in five, but produced nausea, vomiting, and abdominal pain. Two of the four patients who did not respond to erythromycin improved with spiramycin and one with clindamycin but with more severe side effects.

In the prospective study six of the 10 patients treated with erythromycin and all five given spiramycin showed a significant reduction in stool volumes, but in all side effects limited patient tolerance, and after therapy cryptosporidia con- 


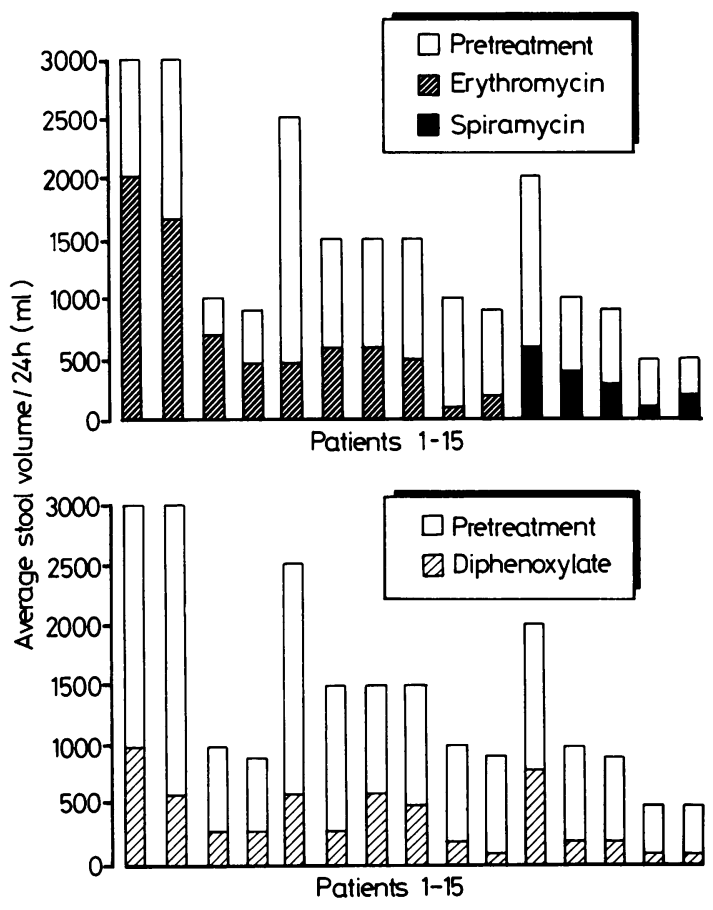

Fig. 4 Volume of diarrhoea in 15 patients pre and post treatment.

tinued to be isolated from the stool (Fig. 4). All patients also showed a significant reduction in stool volumes with diphenoxylate (Fig. 4). The extent of the reduction was proportional to the dose administered but the ability to take the drug was limited because of the development of cramping abdominal pains. In all seven patients given long acting morphine sulphate a reduction of more than $50 \%$ in stool volumes occurred and stools became formed in six patients.

One patient was treated with intravenous somatostatin $(250 \mu \mathrm{g}$ bolus iv over two to three mins in a continuous infusion followed by $3.5 \mu \mathrm{g} / \mathrm{kg} / \mathrm{h}$ over 40 hours, Serono Laboratories [UK] Ltd) for three days. This therapy had no effect on the volume of diarrhoea, but abolished severe cramping abdominal pains which returned on stopping therapy.

Three of our 11 remaining live patients with cryptosporidiosis have received zidovudine (AZT) for more than three months and in all cases the diarrhoea has resolved and cryptosporidia have not been isolated from multiple stool samples (minimum of six/patient).

\section{Discussion}

The frequency of cryptosporidiosis as a complication or presenting feature of AIDS is similar in this study to those from North America but lower than that in African patients studied in Belgium. ${ }^{9}$

The cardinal feature of cryptosporidial infection is severe watery diarrhoea. Up to 171 stools per day has been reported ${ }^{6}$ but in our patients 31 stools per day or less were passed. Admission to hospital and bed rest was reported by the patients in order to reduce the volume and frequency of stools. Abdominal pain and vomiting are unusual presenting features but may occur terminally. All our patients had substantial amounts of weight loss during the course of their illness. The well recognised wasting syndrome (slim disease) in Africa is obviously often related to diarrhoea, but we have certainly seen two patients with constipation who have lost massive amounts of weight and have cryptosporidial infestation of the bowel. Malabsorption is a possible cause for the wasting in these patients, although duodenal biopsies were not normal they did not show severe villous atrophy.

Diagnosis depends primarily on staining of stool samples. We have used the modified Ziehl Neelsen technique which was reported the most reliable in one series. ${ }^{10}$

The numbers of oocysts in the stool may be small and with increasing experience there will be a rise in the frequency of detection of cry'ptosporidia. In the first few years of the AIDS epidemic cryptosporidia were rarely identified in our laboratory, but in recent years have become the predominant stool pathogen in AIDS patients. There has also been a reduction in the mean number of stool samples required before a diagnosis is made. Histology of rectal biopsies yielded a surprisingly low frequency of positive results, although the shizonts were often seen in the duodenal mucosa. Although the shizont does not penetrate the mucosa some of the diarrhoea, malabsorption and weight loss is likely to be the result of small intestinal infestation. Indeed we believe that the controversy of jejunal biopsy abnormalities in AIDS which vary in different reports from severe" to mild ${ }^{12}$ may be caused by coincident cryptosporidial infections in some patients. The seasonal variations in diagnosis of cryptosporidiosis has been observed previously in immunocompetent patients in the $\mathrm{UK}^{13}$ and Canada. ${ }^{14}$

Radiology did not show any diagnostic features of cryptosporidial infection, and we would regard barium meal and follow through, and barium enema as unnecessary in patients with AIDS who have diarrhoea, and cryptosporidia in the stools.

Treatment of this group of patients is well worthwhile. Most of the patients did not die of the direct effects of diarrhoea, and most lead independent useful lives outside the hospital despite continuing 
infection. Although antibiotics do seem to reduce the stool volume presumably by reducing the load of cryptosporidia in the gut, they were unable to eradicate the infection, in our experience in contrast with a previous report of spiramycin. ${ }^{11}$

No subject was able to tolerate the side effects of taking these antibiotics long term, although most of them were prepared to take therapy during exacerbations of the diarrhoea. Our preliminary experience indicated that although spiramycin might help when erythromycin was not beneficial the side effects of abdominal pain and vomiting were particularly severe and this is why more patients were treated with erythromycin in our prospective study. The efficacy of erythromycin was similar to reported results for spiramycin ${ }^{15}$ and spiramycin had a significant effect on the volume of diarrhoea in all patients in which this antibiotic was tried. In view of the side effects our current advice would be to try erythromycin first for one week, and give spiramycin if no effect is observed. In practice antidiarrhoeal agents were the mainstay of therapy and antibiotics and were reserved in our patients for exacerbations of the diarrhoea. Antidiarrhoeal agents were effective in reducing stool volumes in all our patients. This was particularly true of the long acting morphine derivative which frequently resulted in the passage of a solid stool. Such a therapy is not contraindicated in patients with a limited life span and has certainly improved the quality of life considerably in a number of our patients.

Our early results with zidovudine therapy indicates the exciting possibility that the marginal improvement in immune competence induced by this drug may be sufficient to eradicate cryptosporidia from the stool without specific antibiotic therapy. We await the results of our increasing experience and those of others with interest.

\section{References}

1 Tyzzer EE. A sporozoan found in the peptic glands of the common mouse. Proc Soc Exp Biol Med 1907; 5: 12-3.

2 Angus KW. Cryptosporidiosis in man, domestic animals and birds: A review. $J R$ Soc Med 1983; 76: 62-70.

3 Nime FA, Burek JD, Page DL, Hoescher MA, Yardley $\mathrm{JH}$. Acute enterocolitis in a human being infected with the protozoan Cryptosporidium. Gastroenterology 1976; 70: 592-8.

4 Pitlik SD, Faunstein V, Garza D, et al. Human cryptosporidiosis: spectrum of disease. Arch Intern Med 1983; 143: $2269-75$.

5 Jokipii AMM, Hemila M, Jokipii L. Prospective study of acquisition of Cryptosporidium, Giardia Lamplia, and gastrointestinal illness. Lancet 1985; ii: 487-9.

6 Centres for Disease Control. Cryptosporidiosis: assessment of chemotherapy of males with acquired immunodeficiency syndrome (AIDS). MMWR 1982; 31: 589-92.

7 Henriksen SA, Pohlenz JFL. Staining of cryptosporidia by a modified Ziehl-Neelsen technique. Acta Vet Scand 1981; 22: 594-6.

8 Connolly GM, Gazzard BG. Toxic megacolon in cryptosporidiosis. Postgrad Med J 1987; 63: 1103-4.

9 Piot P, Taelman H, Minlangu K B, et al. Acquired immunodeficiency syndrome in a heterosexual population in Zaire. Lancet 1984; ii: 65-9.

10 Garcia LS, Bruckner DA, Brewer TC, Shimizu RY. Techniques for the recovery and identification of Cryptosporidium oocysts from stool specimens. J Clin Microbiol 1983; 18: 185-90.

11 Kotler DP, Gaetz HP, Lange M, Klein EB, Holt PR. Enteropathy associated with the acquired immunodeficiency syndrome. Ann Intern Med 1984; 101: 421-7.

12 Dworkin B, Wormser GP, Rosenthal WS, et al. Gastrointestinal manifestations of the acquired immunodeficiency syndrome: a review of 22 cases. Am J Gastroenterol 1985; 80: 774-8.

13 Baxby D, Hart CA. The incidence of cryptosporidiosis: a two year prospective survey in a children's hospital. J Hyg Camb 1986; 96: 107-11.

14 Montessori GA, Bischoff L. Cryptosporidiosis: a cause of summer diarrhoea in children. Can Med Assoc J 1985; 132: 1285.

15 Fortnoy D, Whiteside ME, Buckley III E, MacLeod CL. Treatment of intestinal cryptosporidiosis with Spiramycin. Ann Intern Med 1984; 101: 202-4. 